\title{
The 2018-19 influenza season in Afghanistan: Epidemiology and Virology
}

\section{Mohammad Nadir Sahak ( $\nabla$ sahak.nadir@gmail.com )}

World Health Organization Country Office for Afghanistan https://orcid.org/0000-0002-0334-3629

\section{Fatima Arifi}

Schiller International University

\section{Aspen A Hammond}

Organisation mondiale de la Sante

Henry J Laurenson-Schafer

Organisation mondiale de la Sante

Sayed Ataullah Saeedzai

Ministry of Public Health

Hafizullah Safi

World Health Organization Country Office for Afghanistan

Abdinasir Abubakar

World Health Organization for Eastern Mediterranean Region

Amgad Elkholy

World Health Organization for Eastern Mediterranean Region

Mohmmad Hafiz Rasooly

Ministry of Public Health

Abdul Nasir Ikram

USAID

\section{Research article}

Keywords: Influenza, Influenza-like illness (ILI), Severe Acute Respiratory Infection (SARI), Seasonal influenza

Posted Date: January 23rd, 2020

DOI: https://doi.org/10.21203/rs.2.21675/v1

License: (c) (i) This work is licensed under a Creative Commons Attribution 4.0 International License. Read Full License 


\section{Abstract}

Objective

This paper aims to provide information on epidemiology and virology of seasonal influenza for the 2018-19 season, and build upon limited information for developing influenza vaccination policy for risk groups in Afghanistan.

Methods

This paper is based on a retrospective analysis of Afghanistan influenza surveillance data, both from influenza-like illness (ILI) and severe acute respiratory infection (SARI) cases during the 2018-19 season. The data for 2018-19 has also been compared to the data of the previous two seasons (2016-2017 and 2017-2018).

Results

During the 2018-19 season, a total number of 10,604 ILI and 5,261 SARI cases were reported to the national influenza sentinel surveillance system. From week 482018 to week 14 2019, a total of 713 ILI specimens were tested for influenza virus, and $10.7 \%$ were positive with majority influenza $A(\mathrm{H} 1 \mathrm{~N} 1)$ pdm09 virus (57\%). Similarly, a total of 1,004 SARI specimens were tested for influenza virus during this period, and $11 \%$ were positive for influenza with the majority caused by influenza $A(H 1 N 1)$ pdm09. Almost two-thirds (62\%) of the ILI-influenza positive cases and $46 \%$ SARI-influenza positive cases aged less than five years. Also, over 50 years old patient was reported to have the second-highest proportion of SARI associated influenza (30\%).

Conclusion

The findings indicate that the seasonal influenza virus results in considerable hospital visits, admissions, and deaths in Afghanistan. The data from this study will inform and guide policymakers in developing national influenza vaccination policies.

\section{Introduction}

Acute Respiratory Infections (ARIs) are the leading cause of death among under-five children in Afghanistan and accounts for $23 \%$ of deaths among this age group [1]. An estimated 750,000 children aged less than five are currently suffering from ARIs annually in Afghanistan [2]. Likewise, national disease surveillance and response (NDSR) data shows that pneumonia had the highest number of deaths (1,924 deaths) compared to other notifiable diseases in 2018 in Afghanistan[3].

Influenza viruses (seasonal and avian) cause acute respiratory infections, ranging from mild to severe cases and even to death, in all parts of the world. The global annual attack rate of influenza is estimated to be $5-10 \%$ among adults and $20-30 \%$ among children. Worldwide, these annual epidemics are estimated to result in about 3 to 5 million cases of severe illness [4]. However, the new estimates show that influenza-related respiratory deaths are likely higher and estimated to be 291,243645,832 (4.0-8.8 per 100,000 individuals) [5].

Epidemics of influenza are causing severe illness and death, especially among children, adults, and people with underlying health conditions. Seasonal influenza is caused by influenza A and influenza B type viruses, which circulate around the world and continues to evolve into new strains [6]. Annual vaccination is recommended at the beginning of each season to groups at risk of severe complications and considered the most effective measure for prevention against seasonal influenza and its complications [7].

A total number of 893 ILI associated influenza cases were reported from 2017 to 2019, with 273 cases in 2017, 326 cases in 2018 and 294 cases in 2019. In the same period, a total of 338 SARI associated influenza cases were reported, with 73 cases in 2017, 124 cases in 2018 and 141 cases in 2019 [8]. 
According to the World Organization for Animal Health, Afghanistan is also a high-risk country for avian influenza as it lies along the migratory pathways of wild birds. The widespread practice of raising poultry in the home to supplement income, low community awareness and a health system that is in the early stages of delivering effective health services make Afghanistan prone to an outbreak of spill-over events of avian influenza [9].

From 2007, the virological surveillance was existing with no epidemiological surveillance. At the beginning of 2014, epidemiological surveillance was added, and lab capacity strengthened through the World Health Organization (WHO) Pandemic Influenza Preparedness (PIP) project. As a result, epidemiological surveillance was instigated in nine hospitals and virological surveillance was revived and integrated. The Central Public Health Laboratory (CPHL) located in the capital city of Kabul, has been recognized as a national influenza center (NIC) since 2009 [10].

Although the epidemiology and impact of influenza are well-defined in developed countries, data, and published papers in developing countries are still limited [11]. Due to the lack of appropriate data on the epidemiology and impact of influenza, influenza control programs, including vaccination, are not a priority in most developing countries [12]. Sufficient influenza epidemiological data is required to guide national vaccine policymakers in developing policy, particularly in low- and middleincome countries with limited resources for healthcare. The published literature about seasonal influenza is missing for the previous years in Afghanistan. Also, there is no influenza vaccination policy in Afghanistan and vaccination is not even provided to high-risk groups including the older population, pregnant women, healthcare providers and people with comorbidities. This paper aims to provide information on epidemiology and virology of influenza for the 2018-19 season and contribute to advocate for developing influenza vaccine policy in Afghanistan.

\section{Methods}

This paper is based on a retrospective analysis of Afghanistan influenza surveillance data, both from influenza-like illness (ILI) and severe acute respiratory infection (SARI) cases during the 2018-19 season, compared to the data of the previous two seasons as both epidemiological and laboratory quality data are available since 2016.

A total of 10,604 ILI and 5,261 SARI cases were reported to the influenza sentinel surveillance system. Information from all ILI and SARI cases (of both sexes and all ages) reported to the influenza surveillance system from week 482018 (commencing 27 November) to week 142019 (ending $30 \mathrm{March}$ ) were included in the study. This period was the influenza season defined by the study.

There are nine surveillance sentinel sites (all are both ILI and SARI ), operating in nine provinces of Afghanistan. Among the sentinel sites, five are regional hospitals, three provincial hospitals and one national hospital (Fig. 1). The criteria for selecting these sentinel sites was representativeness by different geographic region and high population density, which could represent the whole population. The nine SARI and ILI sentinel sites submit specimens and epidemiological data weekly. Data is being entered in EMFLU (regional platform for sharing of epidemiological and virologic data on influenza). Since 2014, all sentinel sites collect throat and nasal swabs from two ILI cases and three SARI cases per week per site selected systematically and send them under the cold chain condition to NIC. The sample collections are increasing by twofold during the influenza season (October to March) (four ILI samples and six SARI cases are being collected per site and per week). Besides, during any suspected outbreak or cluster of ILI or SARI, the surveillance staff collect epidemiologic data and specimens from all cases immediately and share them with surveillance department and the NIC for timely confirmation.

\section{a. Case definition}

Influenza surveillance in Afghanistan uses the WHO case definition of influenza-like illness (ILI), which is "an acute respiratory infection with a measured fever of $\geq 38^{\circ} \mathrm{C}$, and cough, with onset within the past ten days". Similarly, severe acute respiratory infection (SARI) is defined as: "an acute respiratory infection with a history of fever or measured fever of $\geq 38^{\circ} \mathrm{C}$, and cough, with onset within the past ten days, and requiring hospitalization" [13].

b. Laboratory diagnosis

Page 3/17 
All of the laboratory tests for influenza viruses were conducted by CPHL, which is a WHO-designated NIC. The type/subtype of the influenza virus was identified by real-time reverse transcription-polymerase chain reaction (RT-PCR). Influenza clinical specimens and selected influenza virus isolates were sent to the WHO collaborating center in the Centers for Disease Control and Prevention (CDC), Atlanta for antigenic and genetic characterization of the viruses (considering representative samples by age group, severity, and geography). Due to the limited capacity of the NIC, the negative samples for influenza were not tested for other respiratory pathogens.

c. Statistical data analysis

Data were summarized in the form of proportions and frequency tables for all categorical variables. Proportions of ILI and SARI were calculated as the number of ILI cases per 1,000 outpatient consultations and SARI cases per total inpatient admissions. The variables included in the study were ILI and SARI cases, positive influenza and sub-types, demographic characteristics (e.g., sex, age), hospitalization and outcome. Data from EMFLU was exported to SPSS software version 20 for statistical analysis. The epidemic threshold for the proportion of ILI and SARI cases during recent years was defined using the WHO Average curve Shiny app with the two seasons of historical data [14]. As the current WHO Average curve Shiny app was not able to demonstrate the best estimation of the beginning and end week of the season, the Moving Epidemic Method (MEM) 2.15 was used for determining the beginning and end week of the season.

\section{Results}

Influenza-Like Illness (ILI)

The ILI associated consultation rate was above the epidemic threshold from week 1 to 72019 , and overall was lower for the 2018-19 season compared to 2017-18 and 2016-17 seasons (Fig. 2).

From week 48, 2018 to week 14, 2019, a total of 713 ILI specimens were tested for influenza virus, and $(n=76,10.7 \%)$ were positive. Influenza $A(H 1 N 1) p d m 09$ was the major cause, with $(n=43,57 \%)$ positive specimens. It was followed by influenza $A(H 3 N 2)(n=17,22 \%)$ and influenza B (Yamagata lineage) $(n=16,21 \%)$ viruses (Fig. 3). The proportion of ILI-influenza positive peaked at $28 \%$ in January 2019.

Almost two-thirds (62\%) of the ILI-influenza positive cases were aged less than five years old, with $36 \%$ less than two years, and $26 \%$ aged $2-5$ years old. It was followed by by $16 \%$ aged $5-15$ years, $16 \%$ aged $15-50$ years, and $6 \%$ aged over 50 years old. The influenza $\mathrm{A}(\mathrm{H} 1 \mathrm{~N} 1)$ pdm09 virus was more common among under-two children and declining by the increase in age (Fig. 4). Moreover, the ILI associated influenza was higher among males (59\%) than females (41\%) (Table 1). 
Table 1

Demographic characteristics of ILI and positive influenza cases, week 48 2018- week 142019

\begin{tabular}{|llll|}
\hline Characteristic & ILI cases N (\%) & Number of specimens tested & Influenza positive specimens N (\%) \\
\hline Overall & $10,604(100)$ & 713 & $76(100)$ \\
\hline Age group (Year) & $3,080(29)$ & 235 & $27(36)$ \\
\hline 0 to $<2$ & $2,218(21)$ & 156 & $20(26)$ \\
\hline 2 to $<5$ & $1,721(16)$ & 139 & $12(16)$ \\
\hline 5 to $<15$ & $1,723(16)$ & 122 & $12(16)$ \\
\hline 15 to $<50$ & $1,022(10)$ & 49 & $1(1)$ \\
\hline 50 to $<65$ & $840(8)$ & 12 & $4(5)$ \\
\hline$\geq 65$ & & & $45(59)$ \\
\hline Gender & $4,974(47)$ & 347 & $31(41)$ \\
\hline Male & $5,630(53)$ & 366 & \\
\hline Female & & & \\
\hline
\end{tabular}

Severe Acute Respiratory Infection (SARI)

The cumulative number of SARI associated cases were lower for the 2018-19 influenza season compared to 2017-18 and 2016-17 seasons (Fig. 5). However, the proportion of SARI cases per total inpatient admissions was higher during the 2018-19 season compared to 2017-18 but lower than the 2016-17 season. During the 2018-19 season, the SARI cases crossed the epidemic threshold on week 50, 2018, with 264 cases and gradually declined on week 14, 2019, with 217 SARI cases. The SARI admission rate was highest (9.6\%) in week 3 for the 2018-19 season, which was $11.8 \%$ in week 6 for the $2016-17$ season and $8.8 \%$ in week 5 for the 2017-18 season (Fig. 6).

A total number of 128 SARI deaths were reported during week 48, 2018 to week 142019 (CFR $=2.4 \%$ among SARI cases). The proportion of SARI cases that were fatal was lower in the 2018-19 season compared to the 2017-18 season and higher than the 2016-17 season. The peak weeks for death in the 2018-19 season were week $7(n=4, C F R=3 \%)$, week $9(n=5, C F R=4 \%)$ and week $12(n=4, C F R=3 \%)$.

From week 482018 to week 14 2019, a total of 1,004 SARI specimens were tested for influenza virus, and $(n=112,11 \%)$ were positive. More than two-thirds ( $70 \%)$ of SARI associated laboratory-confirmed influenza cases were caused by the influenza $A(H 1 N 1) p d m 09$ virus. It was followed by influenza $A(H 3 N 2) 21(19 \%)$ and then influenza $B$ (Yamagata lineage) $(n=13,12 \%)$. The SARI positivity rate continued to increase from week 49, 2018, and decline from week 12,2019 . Similar to ILI, the proportion of SARI-influenza positive peaked at 23\% in January (week 3 and 4 2019). The influenza A(H1N1)pdm09 was the dominant strain during the seasonal period (Fig. 7).

Almost half (44\%) of SARI reported cases were among under-two children. A large proportion of SARI associated positive influenza cases aged less than five years old (46\%) with $32 \%$ aged less than two years old and $14 \%$ aged $2-5$ years. It was followed by $11 \%$ among $5-15$ years old, $13 \%$ among $15-50$ years old, $12 \%$ among $50-65$ years old and $18 \%$ among $65+$ years old patients (Table 2). The influenza A (H1N1) pdm09 virus was more common among under-two children with 23 positive viruses, and $65+$ years old groups with 12 positive viruses (Fig. 8).

The SARI-associated influenza cases were slightly higher among males (53\%) than females (47\%) (Table 2). 
Table 2

Demographic characteristics of SARI and influenza cases, week 48 2018- week 142019

\begin{tabular}{|llll|}
\hline Characteristics & SARI cases N (\%) & Number of specimens tested & Influenza positive specimens $\mathbf{N}(\%)$ \\
\hline Overall & $5,261(100)$ & 1004 & $112(100)$ \\
\hline Age group (Year) & & & $36(32)$ \\
\hline 0 to $<2$ & $2,331(44)$ & 459 & $16(14)$ \\
\hline 2 to $<5$ & $840(16)$ & 112 & $12(11)$ \\
\hline 5 to $<15$ & $472(9)$ & 77 & $15(13)$ \\
\hline 15 to $<50$ & $474(9)$ & 209 & $13(12)$ \\
\hline 50 to $<65$ & $567(11)$ & 82 & $20(18)$ \\
\hline$\geq 65$ & $577(11)$ & 65 & $59(53)$ \\
\hline Gender & & 567 & $53(47)$ \\
\hline Male & $2,642(50.2)$ & 567 & \\
\hline Female & $2,619(49.78)$ & 437 & \\
\hline
\end{tabular}

\section{Discussion:}

The ILI season was from week 46 to week 10, and from week 48 to week 14 for SARI. The Surveillance 2017 report shows that the influenza season started in October and peaked in December [15]. Afghanistan experiences one peak of influenza season annually based on three years of surveillance data. The influenza-positive cases were highest during January in Afghanistan. This season is similar to other Middle Eastern countries in the region which is in line with the Northern Hemisphere region with large primary peaks between January to March and no or almost undetectable secondary peak [16]. Climatic parameters including temperature, humidity determine influenza season, and rainfalls, both temperate and tropical climate countries, are likely to be the primary driver of influenza seasonality in the Middle East and North Africa [17].

Influenza caused 10,604 outpatient visits, 5,261 SARI admissions (hospitalization), and 128 deaths during the 2018-19 season in Afghanistan. Both SARI and ILI associated rates were lower for the 2018-19 season compared to 2017-18 and 2016-17 seasons. However, SARI cases per total inpatient admissions were higher during the 2018-19 season compared to 2017-18 but lower than the 2016-17 season. Population movement during winter due to conflicts can be a cause of seasonal variation [18] although no specific study has been conducted to determine the variation of influenza cases annually in Afghanistan. Based on the Surveillance data on the burden of influenza in Afghanistan, the NIC has started discussions with related stakeholders to develop the influenza vaccination policy in the country. However, more comprehensive information on the burden of influenza disease among the population is needed to guide the vaccination policy of the country.

A high proportion of influenza-positive cases, SARI (46\%) and ILI (62\%) were reported among under five years old. While only $6 \%$ of ILI influenza-positive cases aged more than 50 years old, a significant proportion of SARI influenza-positive cases aged more than 50 years old (30\%). This is similar to other countries. For example, in New Zealand, the influenza-associated SARI hospitalizations were higher in both young children ( $0-4$ years) and elderly ( $\geq 80$ years) compared to other age groups. Additionally, the influenza-associated ILI disease burden was higher in children aged 1-19 years and young adults (3549 years) compared to other age groups [19].

For both SARI and ILI, the influenza-positive cases higher for males (59\% for ILI and 53\% for SARI) than females ( $41 \%$ for ILI and $47 \%$ for SARI) which can be due to males either being exposed to the outside environment or seeking health care services more than females in Afghanistan. 
Among the total ILI positive cases, more than half of influenza cases were caused by A(H1N1)pdm09 virus (57\%). This was followed by influenza A(H3N2) (22\%) and influenza B (Yamagata lineage) (21\%) viruses. Similarly, among total SARI positive cases, more than two-thirds were caused by influenza $A(\mathrm{H} 1 \mathrm{~N} 1)$ pdm09 $(70 \%)$ virus. It shows that influenza $\mathrm{A}(\mathrm{H} 1 \mathrm{~N} 1) \mathrm{pdm09}$ was the dominant strain during the seasonal period. The most detections occurred in children under two years old. It is similar to other Middle Eastern countries where influenza A has been dominant in most seasons with majority A (H1N1)pdm09 cases during 2016-2018 [20].

During week 482018 to week 14 2019, a total of 713 ILI specimens were tested for influenza virus, and 76(10.7\%) were positive for influenza. In the same period, a total of 1004 SARI specimens were tested for influenza virus, and $112(11 \%)$ were positive for influenza. The low influenza-positive rate might be due to the limited capacity of NIC and epidemiological surveillance. According to the NIC assessment report, there seemed poor compliance with the use of the case definition of SARI and ILI by surveillance staff which is similar to other Middle Eastern countries where clinicians are unaware of the need to use consistent and systematic surveillance case definition of SARI and ILI and enroll only those meeting the case definition (18). The standardized surveillance guidelines and operating procedures at the sites were not readily available. In addition, there was poor cold chain management during sample transportation by local private transport, which may affect the quality of samples received at the NIC (10).

\section{Conclusion:}

During the 2018-19 season, both ILI rate and SARI cumulative numbers were lower compared to the past years; however, the SARI cases per total inpatient admissions were higher compared 2017-18 season. Influenza accounts for a significant proportion of hospital visits, hospitalizations, and deaths in Afghanistan, which highlights the need for protection against the influenza virus and its complications.

Most of the influenza cases are reported among children under-five years old and elderly population of over 50 years. Moreover, the influenza-positive cases both for ILI and SARI were higher among males than females. Influenza A was the dominant virus in Afghanistan with majority influenza $A(\mathrm{H} 1 \mathrm{~N} 1)$ pdm09 virus followed by influenza $A(H 3 N 2)$ and then influenza $B$ (Yamagata lineage) respectively. These findings suggest protective measures against the influenza virus specially among under-five children and older adults. The influenza percent positivity was highest during January in the winter season. This can guide the timing of taking seasonal influenza vaccination shots.

The data from this study is building upon limited epidemiological information for developing influenza vaccination policy among risk groups in Afghanistan. Yet, further detailed epidemiological data, the impact of influenza including socioeconomic impact and influenza burden of disease study are needed to guide a national influenza control policy, including a vaccination strategy in Afghanistan.

\section{List Of Abbreviations}




\begin{tabular}{|ll|}
\hline Acronym & Meaning \\
\hline ARIs & Acute Respiratory Infections \\
\hline CDC & Center for Disease Control and Prevention \\
\hline CFR & Case Fatality Rate \\
\hline CPHL & Central Public Health Laboratory \\
\hline ILI & Influenza-Like Illness \\
\hline MEM & Moving Epidemic Method \\
\hline NIC & National Influenza Center \\
\hline NDSR & National Disease Surveillance and Response \\
\hline PIP & Pandemic Influenza Preparedness \\
\hline RT-PCR & Real-time Reverse Transcription-Polymerase Chain Reaction \\
\hline SARI & Sever Acute Respiratory Infection \\
\hline
\end{tabular}

\section{Declarations}

Ethics approval and consent to participate: This manuscript is based on secondary analysis of Surveillance data, and human subjects were not interviewed or examined for the study. Thus, ethical approval was not required.

Consent for publication: Not applicable

Availability of data and materials: The dataset generated and/or analyzed for the current study are available online in WHO/FluNet and EMFLU. It's also available with the corresponding author on reasonable request.

Competing interests: None of the authors had any financial or non-financial competing interest

Funding: No funding was required for writing the manuscript

Authors' contributions: MNS outlined and initiated the paper, conducted literature review, analysis, drafted the paper and communicated with other co-authors for their feedback and contribution. FA contributed in doing the literature review using her library resources, contributed in data analysis, visualizing the data, interpreting the results reviewed the draft and other versions and provided feedback for improvement. HS contributed in data cleaning, data analysis, reviewing and commenting all versions of the paper. AAH and HJLS provided technical input on analysis of data and determining the influenza season, reviewed all drafts of paper and provided technical inputs for enriching the paper. SAS, AA, AE, MHR and ANI provided technical input on methodology of the paper, data analysis, reviewing the contents of the paper and providing inputs to improve the manuscript.

Acknowledgements: We acknowledge the influenza surveillance sentinel sites focal points for collecting the epidemiological and specimen collection data and the staff at the national influenza center for doing the laboratory tests. We also acknowledge PIP Partnership Contribution as a source of funding support for the influenza surveillance project in Afghanistan.

Corresponding Authors' information

Dr. Mohammad Nadir Sahak is working as program manager for the Infectious Hazard Management unit of WHO country office in Afghanistan. He is also the focal point for the pandemic influenza preparedness project in Afghanistan. Having an MD and MSc in Epidemiology and Biostatistics, his research areas mostly focused on infectious diseases including influenza, Crimean-Congo hemorrhagic fever and other emerging and re-emerging diseases. 


\section{References}

1. Ministry of Public Health, Central Statistics Organization, ICF Macro, Indian Institute of Health Management Research, World Health Organization Regional Office for the Eastern Mediterranean. Afghanistan Mortality Survey 2010. Calverton, Maryland, USA: APHI/MoPH, CSO, ICF Macro, IIHMR and WHO/EMRO; 2011.

2. 2015 Afghanistan Humanitarian Needs Overview - Afghanistan [Internet]. [cited Sep 12, 2019]. Available from: https://reliefweb.int/report/afghanistan/2015-afghanistan-humanitarian-needs-overview.

3. Ministry of Public Health. National Disease Surveillance and Response Annual Report (2018). In press 2018.

4. Mahima Venkatesh, Charles R Doarn, Mark Steinhoff, Jun Ying. Assessment of burden of seasonal influenza in India and consideration of vaccination policy. Global Journal of Medicine and Public Health. 2016;5(5):1-10.

5. Iuliano AD, Roguski KM, Chang HH, Muscatello DJ, Palekar R, Tempia S, et al. Estimates of global seasonal influenzaassociated respiratory mortality: a modelling study. The Lancet. 2018 /03/31;391(10127):1285-300.

6. Drăgănescu A, Săndulescu O, Florea D, Vlaicu O, Streinu-Cercel A, Oțelea D, et al. The 2017-2018 influenza season in Bucharest, Romania: epidemiology and characteristics of hospital admissions for influenza-like illness. BMC Infect Dis. 2019 Nov 12;19(1):967.

7. Influenza (Seasonal) [Internet]. [cited Dec 29, 2019]. Available from: https://www.who.int/news-room/factsheets/detail/influenza-(seasonal).

8. Pathogen distribution of positive cases. [Internet].: WHO/EMRO; 2019 [cited January 5, 2019]. Available from: http://emflu.emro.who.int/default.aspx.

9. Highly pathogenic avian influenza, Afghanistan. [Internet].; 2018 [cited June 12, 2019]. Available from: https://www.oie.int/wahis_2/public/wahid.php/Reviewreport/Review?page_refer=MapFullEventReport\&reportid=25772.

10. Rasooly MH, Sahak MN, Saeed KI, Krishnan SK, Khan W, Hassounah S. Capacity of the national influenza surveillance system in Afghanistan, a chronic conflict setting. Eastern Mediterranean health journal = La revue de sante de la Mediterranee orientale = al-Majallah al-sihhiyah li-sharq al-mutawassit. 2016 Oct 2;22(7):476-81.

11. Matheka DM, Mokaya J, Maritim M. Overview of influenza virus infections in Kenya: past, present and future. Pan Afr Med J. $2013-4-08 ; 14(138)$.

12. Nguyen YT, Graitcer SB, Nguyen TH, Tran DN, Pham TD, Le MTQ, et al. National surveillance for influenza and influenzalike illness in Vietnam, 2006-2010. Vaccine. 2013 Sep 13,31(40):4368-74.

13. Global Epidemiological Surveillance Standards for Influenza; [Internet].: WHO; 2013 [cited 15 June 2019]. Available from: https://www.who.int/influenza/resources/documents/WHO_Epidemiological_Influenza_Surveillance_Standards_2014.pdf.

14. WHO Average Curve; [Internet].: WHO; 2019 [cited 15 Aug 2019]. Available from: https://whoinfluenza.shinyapps.io/averagecurves/.

15. Ministry of Public Health. National Disease Surveillance and Response Annual Report 2017. In press 2018.

16. Caini S, El-Guerche Séblain C, Ciblak MA, Paget J. Epidemiology of seasonal influenza in the Middle East and North Africa regions, 2010-2016: Circulating influenza A and B viruses and spatial timing of epidemics. Influenza and Other Respiratory Viruses. 2018 May;12(3):344-52.

17. Tamerius JD, Shaman J, Alonso WJ, Alonso WJ, Bloom-Feshbach K, Uejio CK, et al. Environmental Predictors of Seasonal Influenza Epidemics across Temperate and Tropical Climates. PLoS pathogens. 2013 Mar;9(3):e1003194.

18. Abubakar A, Barakat A, Ahmed A, El Kholy A, Alsawalh L, Al Ariqi L, et al. Fourth meeting of the Eastern Mediterranean Acute Respiratory Infection Surveillance (EMARIS) network and first scientific conference on acute respiratory infections in the Eastern Mediterranean Region, 11-14 December, 2017, Amman, Jordan. Journal of Infection and Public Health. 2019 Jul;12(4):534-9.

19. WHO National Influenza Centre, Health Intelligence Team, Institute of Environmental Science and Research. The New Zealand influenza data in 2018. Science for Communities; 24-27 September 2018.; Atlanta, USA: ; Aug 31, 2018. 
20. Caini S, El-Guerche Séblain C, Ciblak MA, Paget J. Epidemiology of seasonal influenza in the Middle East and North Africa regions, 2010-2016: Circulating influenza A and B viruses and spatial timing of epidemics. Influenza Other Respir Viruses. 2018 05;12(3):344-52.

\section{Figures}

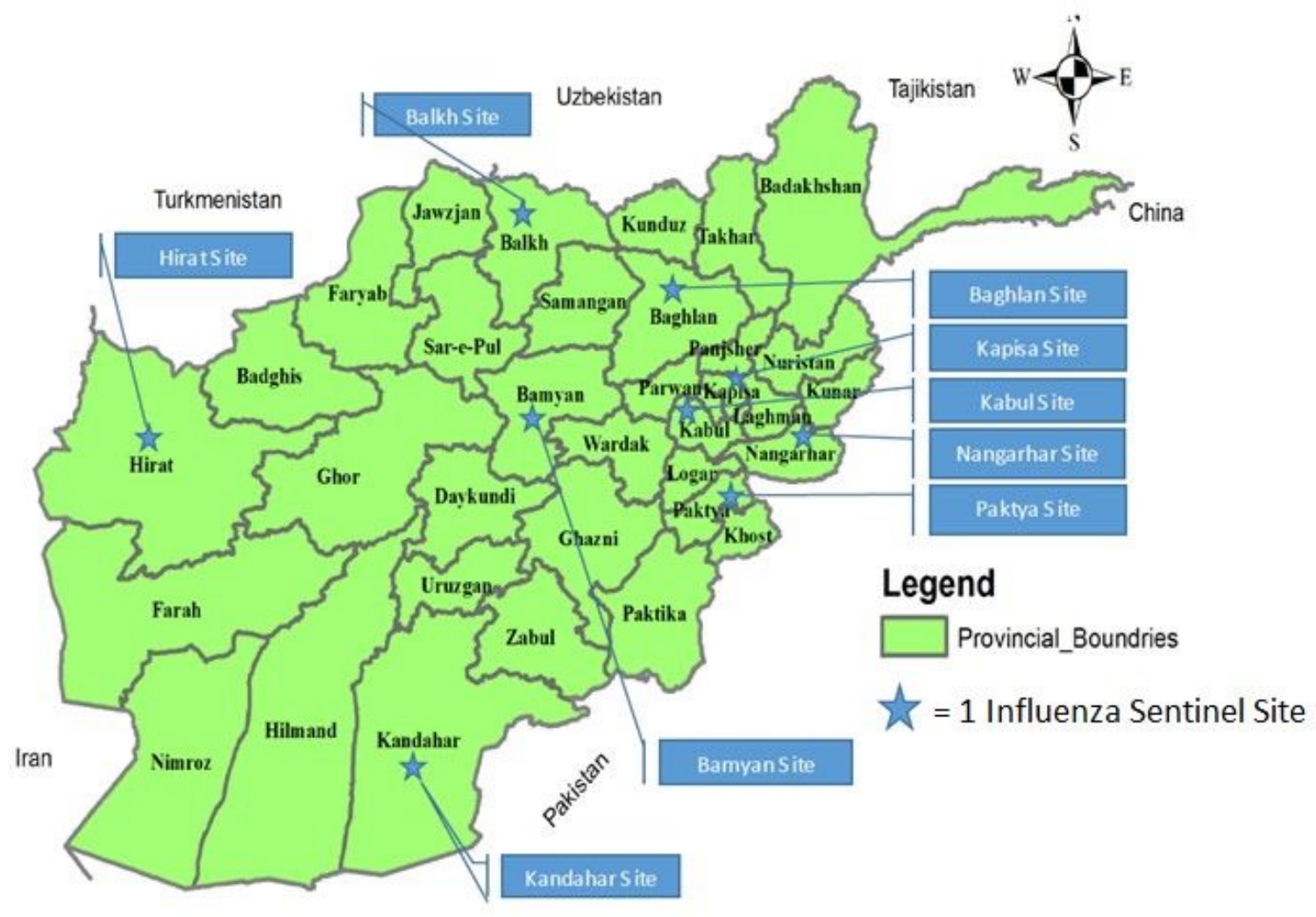

\section{Figure 1}

Influenza surveillance sentinel sites in Afghanistan. Figure 1 shows the nine SARI/ILI sentinel sites geographical distribution in Afghanistan. 


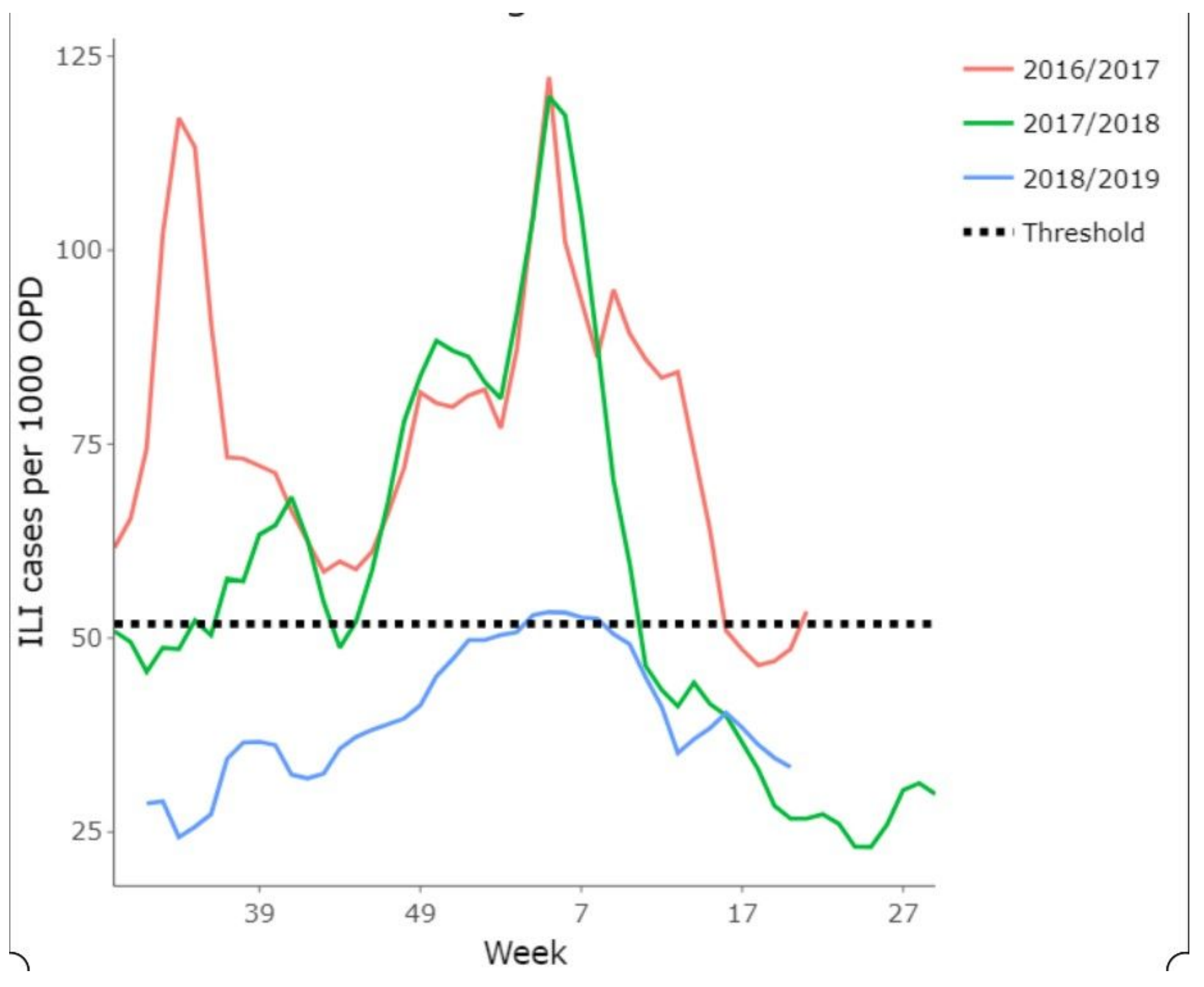

Figure 2

Weekly ILI consultation rate in 2018-19 compared to 2016-2018 seasons. Figure 2 shows the ILI consultation rate per 1000 outpatient consultations in three seasons. The peaks are aligned for each season. The horizontal line is the median calculated threshold. The blue line is ILI consultation rate in the 2018-19 season , green line for 2017-18 and orange line for the 2016-17 season. The horizontal axis is the week number and the vertical axis is ILI consultation per 1000 outpatient consultations. 


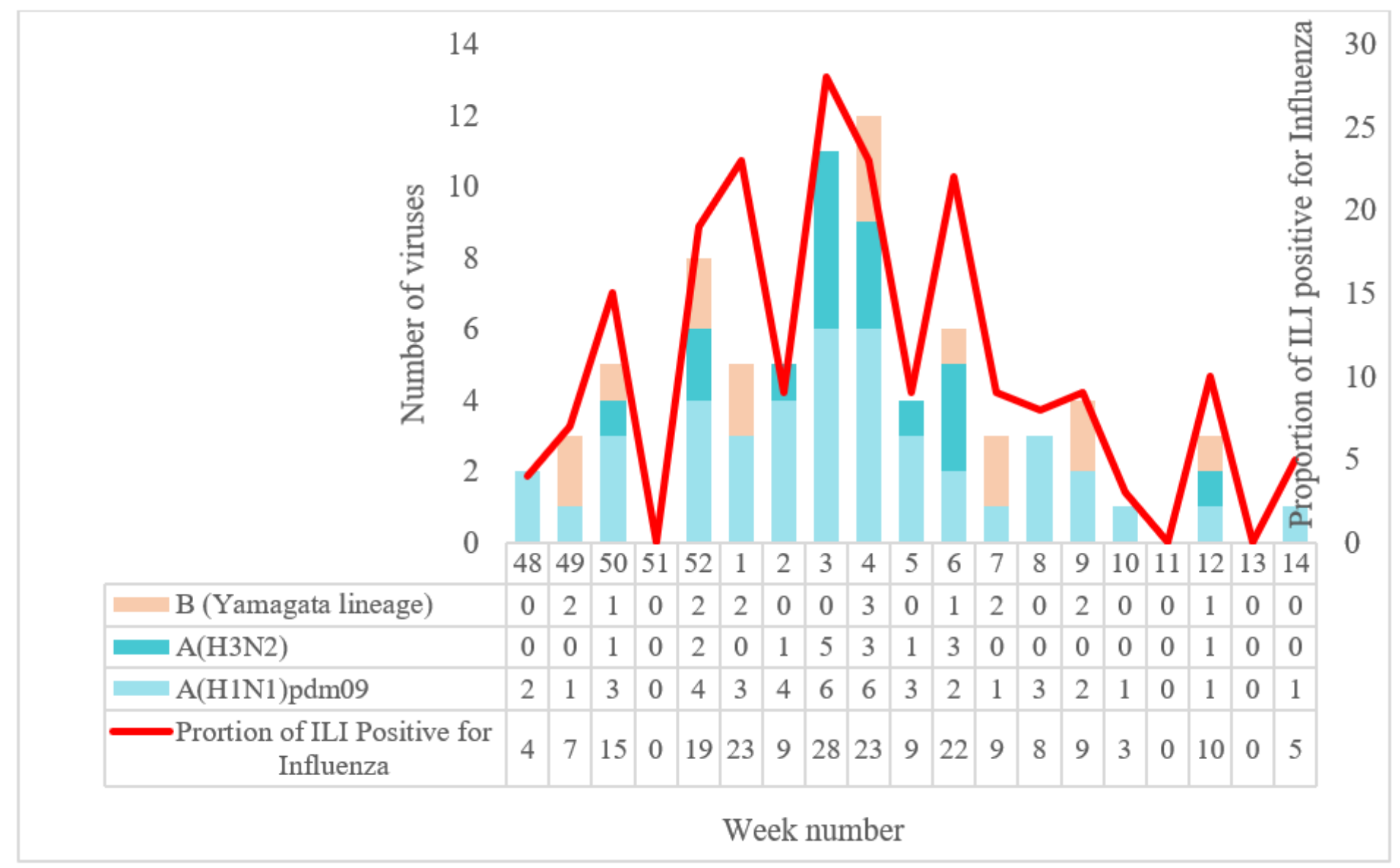

\section{Figure 3}

Temporal distribution of the number and proportion of influenza viruses from ILI specimens, by type and week. Figure 3 shows the number of influenza viruses (vertical axis) type from ILI specimens during seasonal weeks (horizontal axis) in which the $A(H 1 N 1) p d m 09$ is the predominant virus during the seasonal weeks. The red line chart shows proportion of influenza positive on the right vertical axis. 


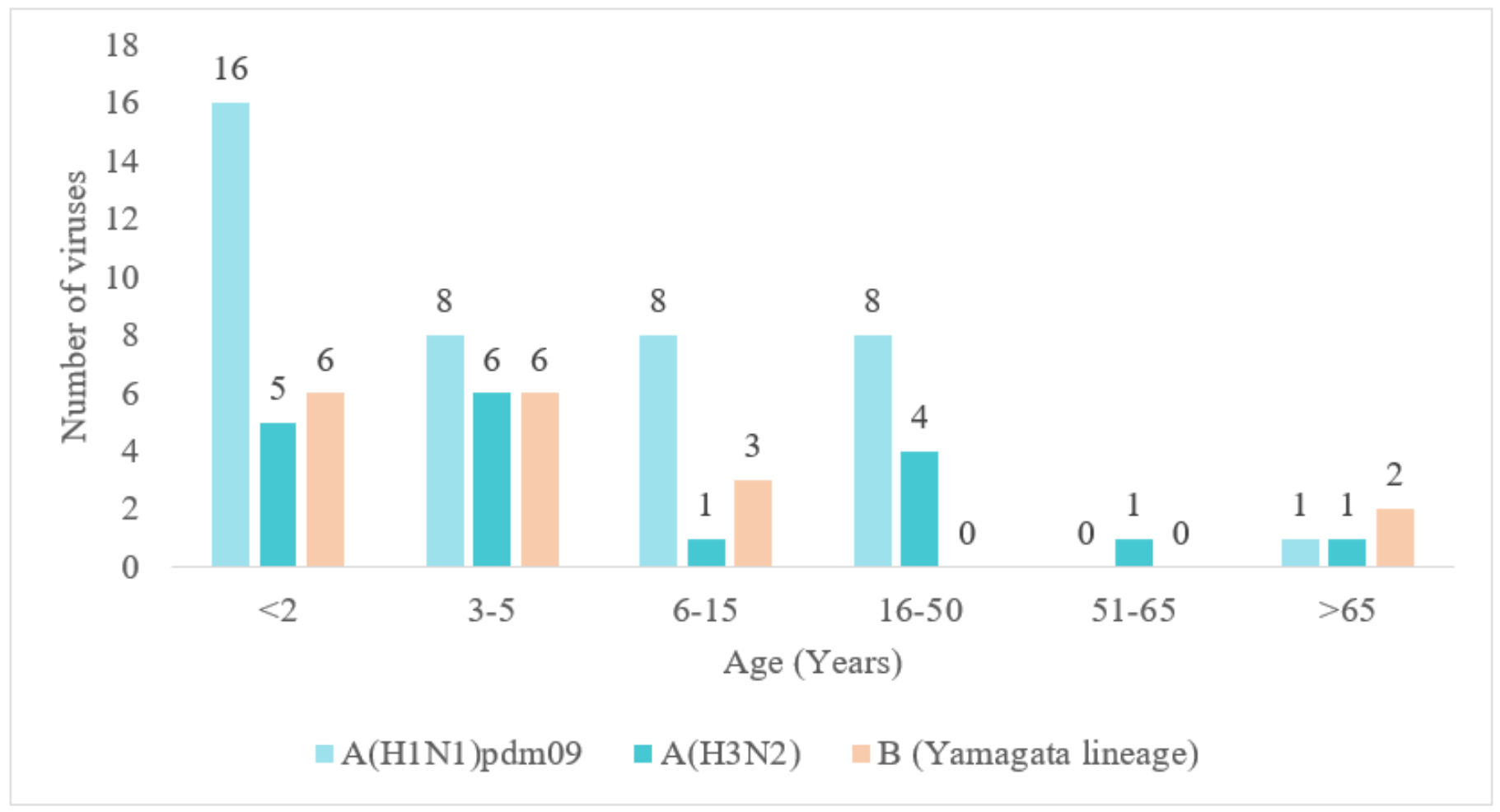

\section{Figure 4}

Distribution of Influenza virus subtype by age group from ILI positive cases, week 48 2018-week 142019 . Figure 4 shows the distribution of influenza virus by age group. The vertical axis shows six categories of age groups and the vertical axis shows the number of influenza viruses by type. 


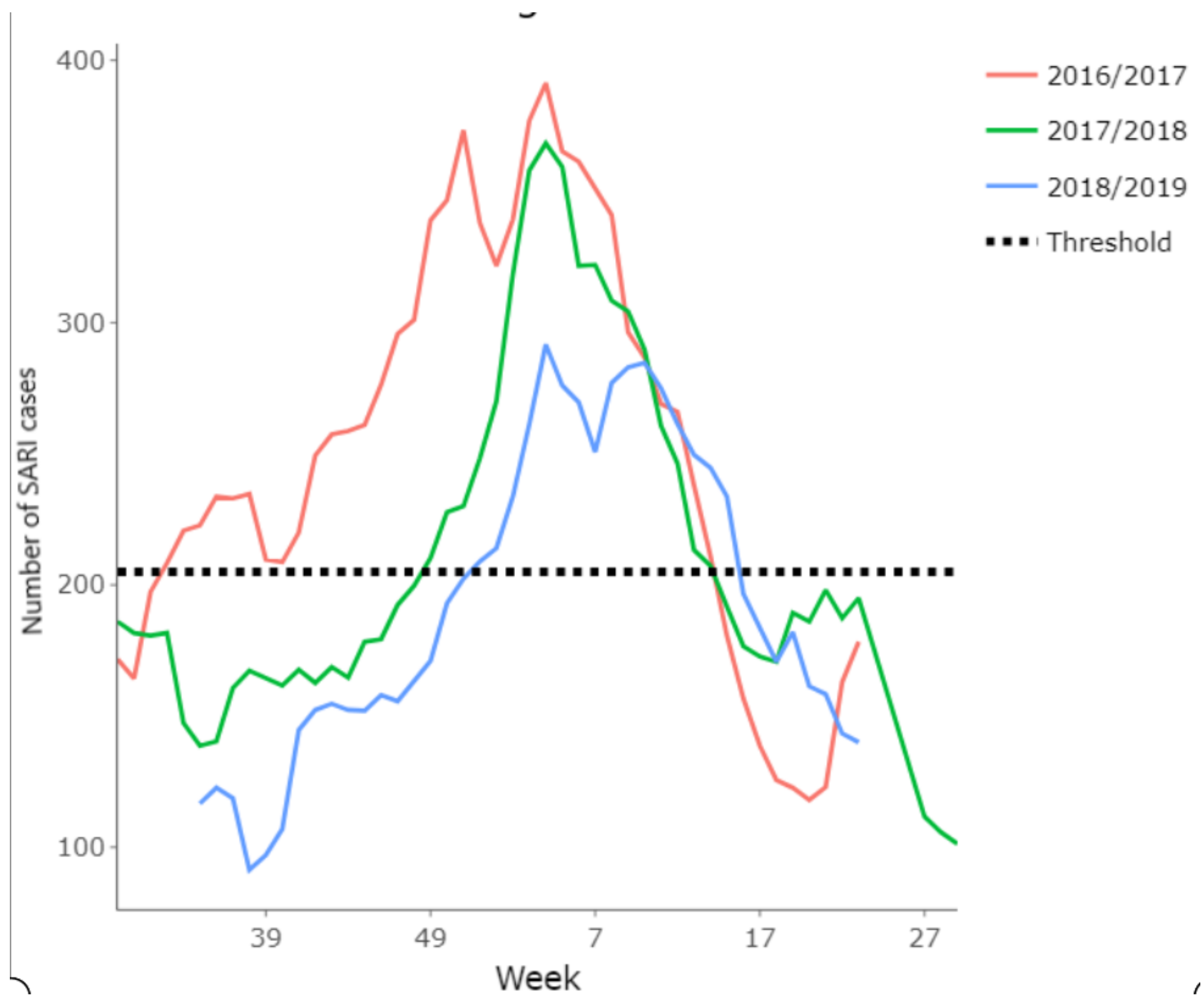

Figure 5

Number of SARI cases in 2018-19 compared to 2016-18 season. Figure 5 shows the number of SARI cases from 2016-17 to 2018-19 seasons using WHO Shinnyapp analysis. The horizontal axis shows the week number and vertical axis shows the number of SARI cases. 


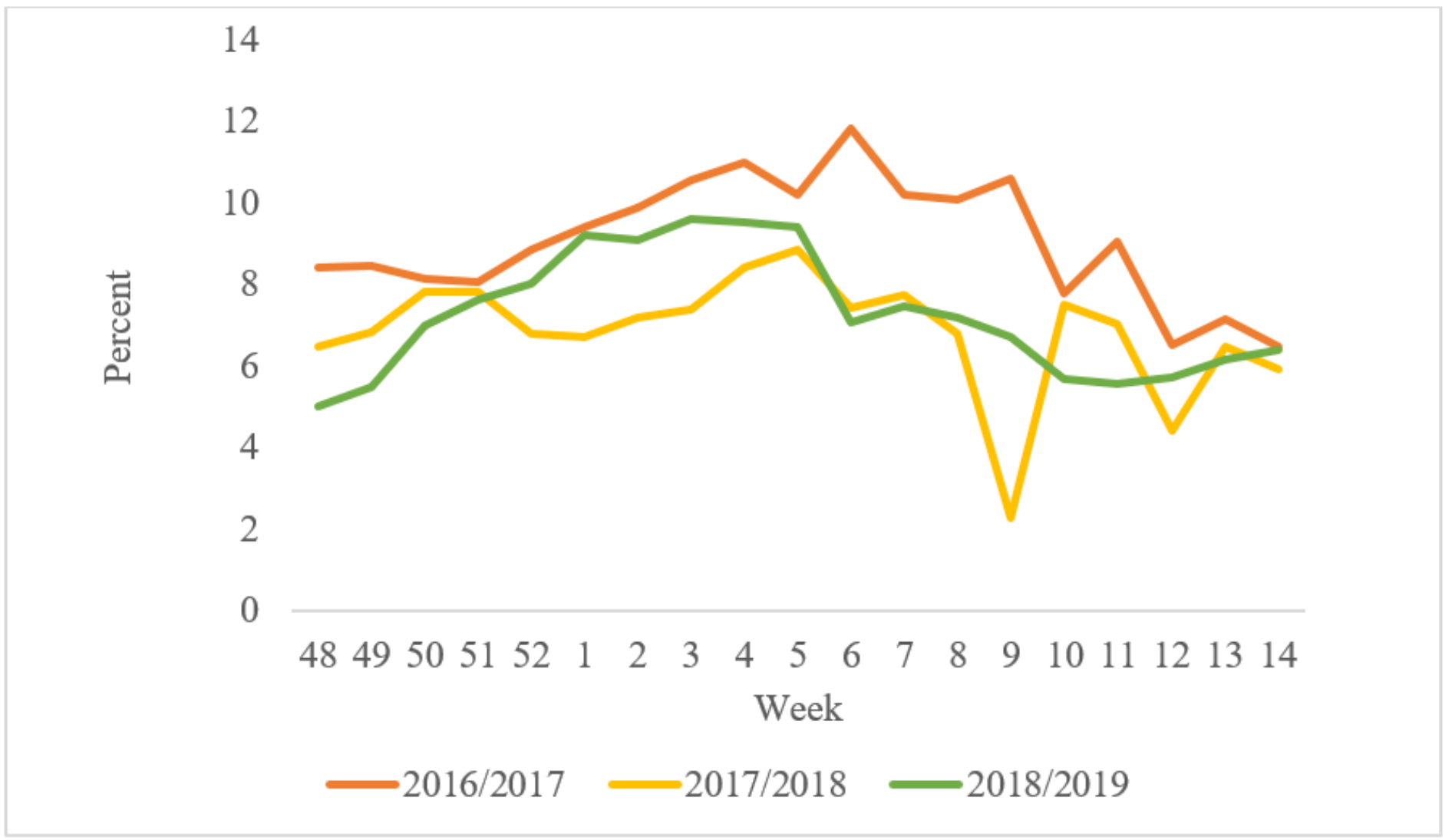

\section{Figure 6}

Proportion of SARI cases out of total inpatient admissions, in 2018-19 compared to 2016-18 seasons. Figure 6 shows the proportion of SARI cases per total inpatient admissions in 2018-19 season compared to 2017-18 and 2016-17 seasons during the seasonal weeks. The horizontal axis shows the week number and the vertical axis shows the percentage of SARI cases per total inpatient admissions. 


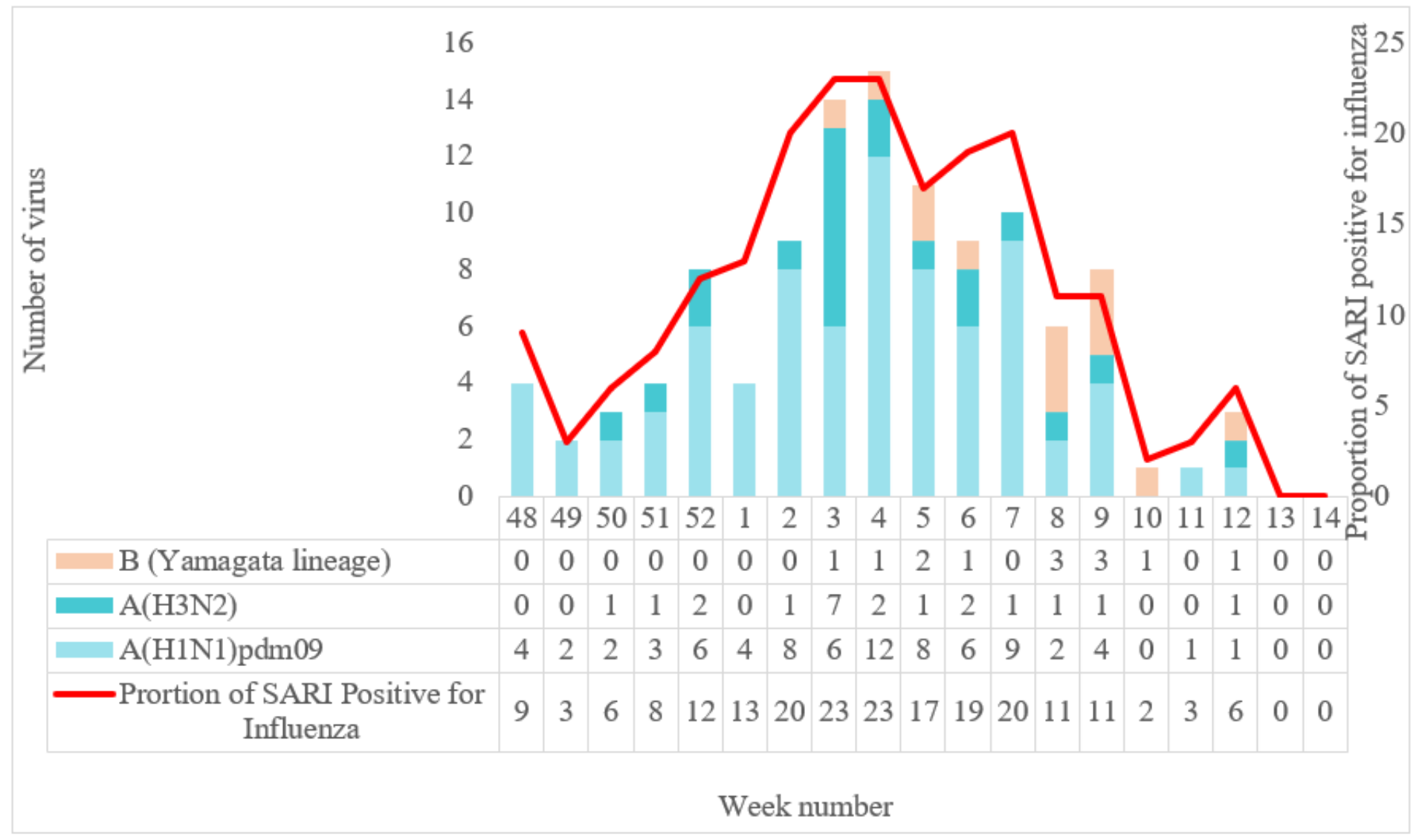

\section{Figure 7}

Temporal distribution of the number and proportion of influenza viruses from SARI specimens, by type and week. Figure 7 shows the number of influenza viruses type from SARI specimens during seasonal weeks. The horizontal axis is the week number and the left vertical axis is the number of viruses. The red line chart shows proportion of influenza positive on the right vertical axis. 


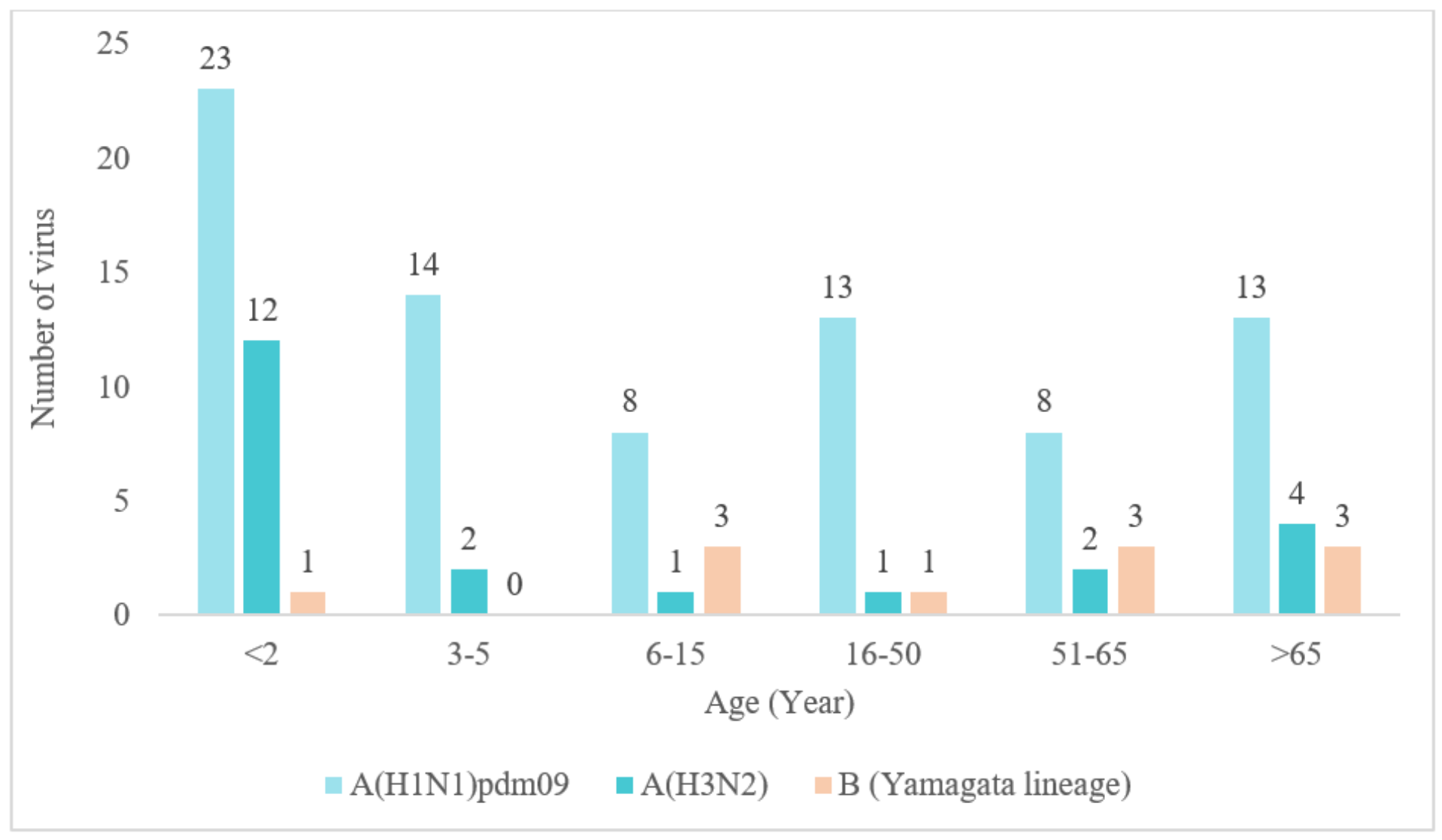

\section{Figure 8}

Distribution of virus by age group from SARI positive cases, week 48 2018-week 14 2019. Figure 8 shows the number of viruses from SARI specimen by age group. The horizontal axis is the six categories of age group and the vertical axis the number of viruses. 